\title{
Intestinal T-cell lymphoma, NOS, presenting with sole peritoneal and mucosal lymphomatosis throughout abdominal cavity
}

Keywords: intestinal T-cell lymphoma NOS, peritoneum, peritoneal lymphomatosis, intestinal mucosa, lymphoepithelial lesion

\section{CASE REPORT}

A 54-year-old man without a history of enteropathy developed fever, nausea, anorexia, and abdominal pain one month prior to his initial hospital visit. He was tentatively diagnosed with peritonitis carcinomatosa, and emergent laparotomy was performed in a regional hospital. Intraoperatively, the abdominal cavity was filled with diffusively thickened serous membranes and mesenteries. No identifiable mass, lymph node swelling, or hepatosplenomegaly was noted. Pathological examination of a peritoneal specimen revealed malignant lymphoma of the diffuse small-cell type with inconclusive immunophenotyping.

After the operation, he was transferred to our hospital. Although the abdomen was taut and distended, abdominal pain was moderate without rebound tenderness or signs of intestinal obstruction on admission. Superficial lymphadenopathy was not noted. His consciousness was alert without neurological abnormalities. Abdominal CT showed diffusively thickened serous membranes, and it was difficult to identify the intestinal configuration (Figure 1).

The white cell count was $20 \times 10^{9} / \mathrm{L}$ with $4 \%$ myelocytes,

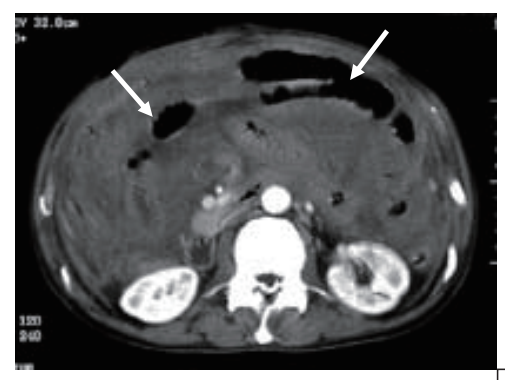

Kidney level
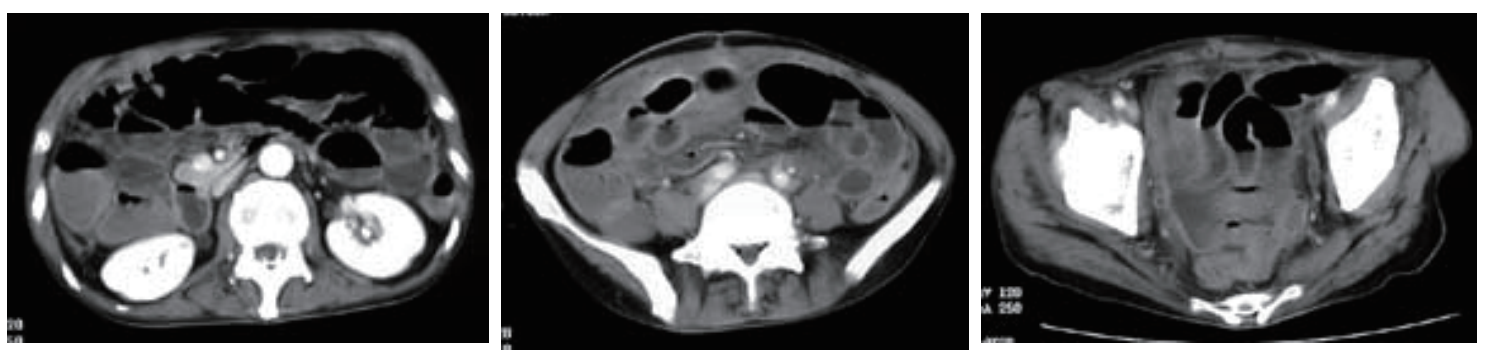

During chemotherapy: 2 months later

Fig. 1. Contrast $\mathrm{CT}$ of the abdomen before and after chemotherapy.

Upper column: Imaging before chemotherapy. The abdominal cavity was filled with thickened serous membranes and mesenteries, and all intestines were embedded in these membranes with an unclear intestinal configuration. Tumor formation was not noted. Arrows indicate markedly enlarged intestines filled with air (not free air). The amount of ascites was modest.

Lower column: Imaging after chemotherapy. The thickening of the serous membranes was reduced and the outlines of individual intestines could be visualized with slightly thickened intestinal walls. 
$77 \%$ neutrophils, $1 \%$ basophils, 5\% lymphocytes, and $13 \%$ monocytes, a hemoglobin concentration of $11.1 \mathrm{~g} / \mathrm{dL}$, and a platelet count of $471 \times 10^{9} / \mathrm{L}$. Abnormal lymphocytes were not observed in the peripheral blood or bone marrow. Serum LDH was as high as $609 \mathrm{IU} / \mathrm{L}$ (normally below 450), CRP was also elevated to $4.2 \mathrm{mg} / \mathrm{dL}$ (normally below 0.5 ), and soluble interleukin-2 receptor was as high as $8,210 \mathrm{U} / \mathrm{mL}$ (normally 135-483). Serum tumor markers including CEA, CA19-9, and $\alpha$-fetoprotein were all within normal limits. Serologic tests for HBV, HCV, HTLV-1, and HIV yielded negative results. Serologic tests for EBV showed a previous infection pattern. Plasma PCR analysis for HHV-8 gave a negative result. We could not perform colonoscopy to obtain a biopsy specimen because of his poor condition.

The growth of the lymphoma was rapid, and we treated him with intensity-reduced CHOP. After 3 courses of treatment that spanned 8 weeks, his abdomen became soft and flat. The outline of the individual intestines could be visualized on CT (Figure 1). However, the effect of treatment was transient, and he developed massive watery stools with a dark-green color. We treated him with salvage chemotherapy (etoposide and vincristine) without improvement, and he died 4 months after admission.

At autopsy, the intestines adhered together with thickening of the peritoneum and mesenteries throughout the abdominal cavity, and it was difficult to separate them. There were no mass lesions or enlarged lymph nodes. Haustra of the large intestine had completely disappeared. The macroscopic pathologic diagnosis was extensive neoplastic peritonitis without an identifiable primary lesion because of diffuse disease. Macroscopic features of the colon showed patchy fibrous thickening of serosal membranes and an edematous intestinal wall (Figure 2). Microscopically, marked infiltrations of lymphoma cells were observed in the peritoneum, mesenteries, mucosa of the small and large intestines, and pleural serous membrane, as shown in Figure 3 from the large intestine, in which the infiltration of abnormal lymphocytes was conspicuous in the mucosa and subserosa (Figure 3). This invasion was massive and space-occupying (Figure 4A/B). Lymphoma cells were small and some of them showed nuclear atypism (Figures 4A/5A). Immunohistochemical

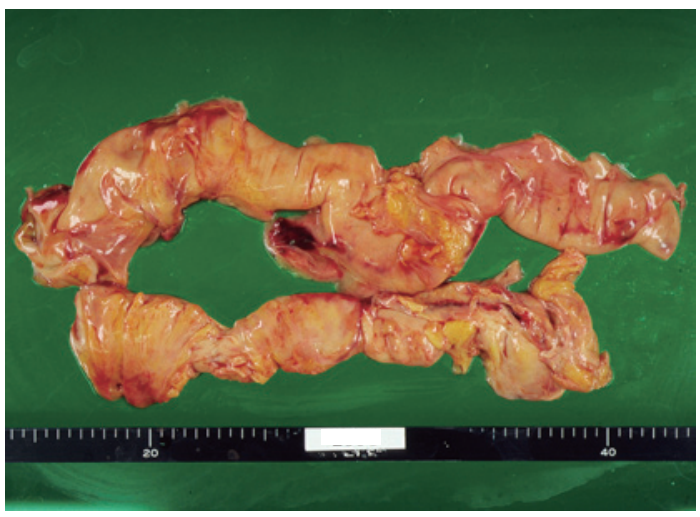

Fig. 2. Macroscopic features of the colon at autopsy. The serosal membrane showed patchy fibrous thickening, and the intestinal wall was edematous. examination demonstrated that these cells expressed CD45RA/RO, CD3, CD4 (faintly), CD5, and CD7 but not CD20, CD8, CD10, CD56, or perforin 1 (Figure 5; data not shown for CD45RA/RO, CD5, CD7, CD10, and perforin 1). Residual crypts were intact without epitheliotropism by lymphoma cells (Figure 4B). The lymphoma cells were negative for EBER (in situ hybridization), LMP-1, and HHV-8 (by PCR). Furthermore, PCR demonstrated monoclonal rearrangement of the TCR- $\beta$ gene. Lymphomatous infiltration was not observed in the liver, spleen, kidney, pancreas, or bone marrow. Thus, the final pathologic diagnosis was intestinal T-cell lymphoma, NOS, extensively invading the peritoneum, mesenteries, and intestinal mucosa throughout the abdominal cavity.

The tumoral invasion in the present patient as peritoneal lyphomatosis ${ }^{1-4}$ is extremely rare in terms of infiltration of all the peritoneum and intestinal mucosa in the abdominal cavity without tumor formation or invasion to other organs. It should be emphasized that extensive disease was observed at the time of presentation of the patient but not in an advanced stage. To the best of our knowledge, there have been only 2 patients similar to ours reported in the literature: one with primary effusion lymphoma (B-cell lineage) ${ }^{5}$ and the other with Burkitt-like B-cell lymphoma. ${ }^{6}$ Therefore, T-cell lymphoma exhibiting this pattern of lymphoma invasion has not been reported. Indeed, B-cell lymphoma, especially DLBCL, has been most frequently reported in peritoneal lymphomatosis. ${ }^{3,4}$

The 2017 WHO classification ${ }^{7}$ includes 4 subtypes of intestinal T-cell lymphoma. Regarding the lymphoma

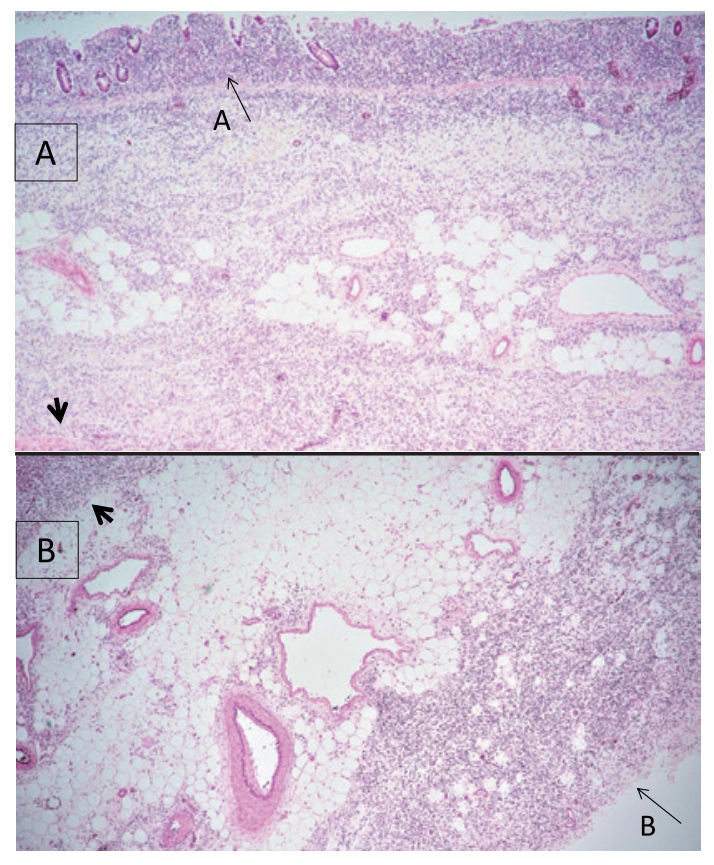

Fig. 3. Microscopic examinations at low magnification of the colon revealed that atypical lymphoid cells infiltrated the whole intestinal wall layer, particularly in the mucosa and subserosa. Arrows $\boldsymbol{A}$ and $\boldsymbol{B}$ indicate the mucosa and subserosa, respectively (H-E staining, $\times 20$ ). Arrowheads indicate the muscular layer. 


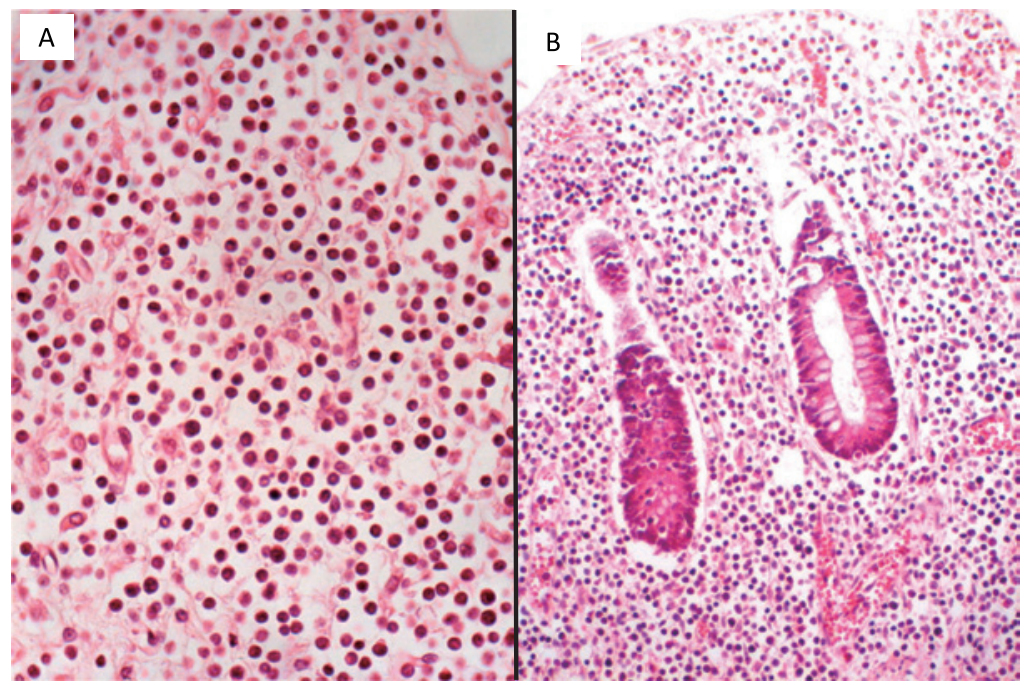

Fig. 4. Histopathological examination of the peritoneum and colonal mucosa at autopsy.

$\boldsymbol{A}$ : The peritoneum was diffusely infiltrated by small lymphoid cells. These cells had round nuclei with slight atypism and a high N/C ratio. H-E staining, $\times 400$. $\boldsymbol{B}$ : The colonic mucosa was also densely infiltrated by the same small atypical lymphoid cells. Epitheliotropism of these lymphoid cells was not observed. H-E staining, $\times 200$.

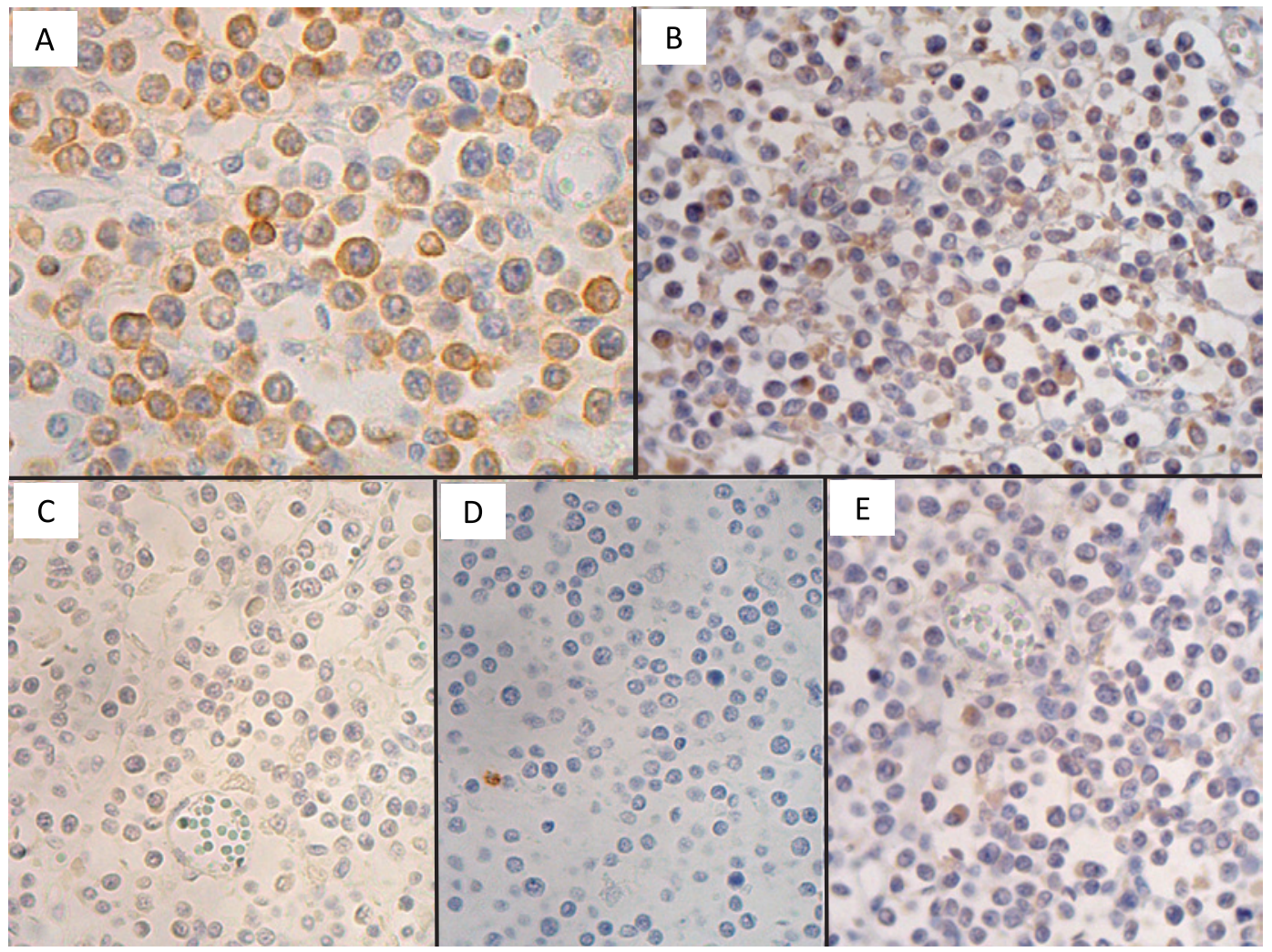

Fig. 5. Immunohistochemical examination of the peritoneum and colonal mucosa at autopsy.

These abnormal lymphoid cells were positive for CD3 $(\boldsymbol{A})$ and CD4 (faintly) $(\boldsymbol{B})$ but negative for CD20 $(\boldsymbol{C})$, CD8 $(\boldsymbol{D})$, and CD56 $(\boldsymbol{E})(\times 400$ for CD3 and $\times 200$ for the rest $)$. 
subtype of the present patient, intestinal T-cell lymphoma, NOS, may be reasonable, considering the absence of a history of enteropathy, aggressive clinical course, small-sized lymphoma cells, no epitheliotropism, and immunophenotype of lymphoma cells $\left(\mathrm{CD}^{+}, \mathrm{CD} 4^{ \pm}, \mathrm{CD}^{+}, \mathrm{CD}^{+}, \mathrm{CD} 8^{-}, \mathrm{CD} 56^{-}\right.$, LMP-1').

We also searched for whether sole peritoneal invasion similar to ours has been described in the literature focusing on intestinal T-cell lymphoma. ${ }^{7-15}$ Consequently, the localization of intestinal T-cell lymphoma at presentation was revealed to be generally regional ${ }^{7-15}$ and cases with extensive disease like the present case could not be found even in this way. Because intestinal T-cell lymphoma, NOS, is currently not considered a specific disease entity, ${ }^{7}$ it is difficult to distinguish the present type of lymphoma from peripheral T-cell lymphoma, NOS, arising in the peritoneum; however, the cellular origin of this type of lymphoma will be clarified in the future because of the marked affinity of the tumor for the peritoneum and mucosa.

\section{ACKNOWLEDGMENTS}

The authors are grateful to Miss Mizue Higashi for her excellent support of manuscript preparation and the literature search.

\section{CONFLICT OF INTEREST}

The authors declare that there is no conflict of interest regarding this study.

\section{REFERENCES}

1 Runyon BA, Hoefs JC. Peritoneal lymphomatosis with ascites. A characterization. Arch Intern Med. 1986; 146 : 887-888.

2 Lynch MA, Cho KC, Jeffrey RB Jr, Alterman DD, Federle MP. CT of peritoneal lymphomatosis. AJR Am J Roentgenol. 1988; $151: 713-715$.

3 Kim YS, Cho OK, Song SY, et al. Peritoneal lymphomatosis: CT findings. Abdom Imaging. 1998; 23 : 87-90.

4 Karaosmanoglu D, Karcaaltincaba M, Oguz B, et al. CT findings of lymphoma with peritoneal, omental and mesenteric involvement: peritoneal lymphomatosis. Eur J Radiol. 2009; 71 :313-317.

5 Tanaka S, Katano H, Tsukamoto K, et al. HHV8-negative primary effusion lymphoma of the peritoneal cavity presenting with a distinct immunohistochemical phenotype. Pathol Int. 2001; $51: 293-300$.

6 Yapar AF, Reyhan M. 18F-FDG uptake in diffuse peritoneal lymphomatosis. Clin Nucl Med. 2012; 37 : e176-e177.

7 Jaffe ES, Chott A, Ott G, et al. Intestinal T-cell lymphoma. In : Swerdlow SH, Campo E, Harris NL, et al. (eds) : WHO Classification of Tumours of Haematopoietic and Lymphoid Tissues. 4th ed, Lyon, IARC, World Health Organization. 2017; pp. 372-380.

8 Gale J, Simmonds PD, Mead GM, Sweetenham JW, Wright DH. Enteropathy-type intestinal T-cell lymphoma: clinical features and treatment of 31 patients in a single center. J Clin Oncol. $2000 ; 18: 795-803$.

9 The Non-Hodgkin's Lymphoma Classification Project. A clinical evaluation of the International Lymphoma Study Group classification of non-Hodgkin's lymphoma. Blood. 1997; 89 : 3909-3918.

10 Delabie J, Holte H, Vose JM, et al. Enteropathy-associated T-cell lymphoma: clinical and histological findings from the international peripheral T-cell lymphoma project. Blood. 2011; $118: 148-155$.

11 Malamut G, Chandesris O, Verkarre V, et al. Enteropathy associated T cell lymphoma in celiac disease: A large retrospective study. Dig Liver Dis. 2013; 45 : 377-384.

12 Tse E, Gill H, Loong F, et al. Type II enteropathy-associated T-cell lymphoma: a multicenter analysis from the Asia Lymphoma Study Group. Am J Hematol. 2012; 87 : 663-668.

13 Attygalle AD, Cabeçadas J, Gaulard P, et al. Peripheral T-cell and NK-cell lymphomas and their mimics; taking a step forward - report on the lymphoma workshop of the XVIth meeting of the European Association for Haematopathology and the Society for Hematopathology. Histopathology. 2014; 64 : 171-199.

14 Perry AM, Warnke RA, Hu Q, et al. Indolent T-cell lymphoproliferative disease of the gastrointestinal tract. Blood. 2013; 122 : 3599-3606.

15 Egawa N, Fukayama M, Kawaguchi K, et al. Relapsing oral and colonic ulcers with monoclonal T-cell infiltration. A low grade mucosal T-lymphoproliferative disease of the digestive tract. Cancer. 1995; $75: 1728-1733$.

\section{Naoko Watanabe-Okochi, ${ }^{1)}$ Yukihiro Imai, ${ }^{2)}$ Hayato Kimura, ${ }^{3)}$ Daisuke Yamashita, ${ }^{2)}$ Shigeo Hara, ${ }^{2)}$ Takayuki Takahashi')}

${ }^{1)}$ Departments of Hematology and Clinical Immunology, Kobe City Medical Center General Hospital, Kobe, Japan, ${ }^{2}$ Department of Pathology, Kobe City Medical Center General Hospital, Kobe, Japan, ${ }^{3)}$ Department of Diagnostic Pathology, Itami City Hospital, Itami, Hyogo, Japan. Naoko Watanabe-Okochi is currently at The Laboratory Department, Kanto-Koshinetsu Block Blood Center, Japanese Red Cross, Tokyo, Japan.

Yukihiro Imai is currently at the Department of Diagnostic Pathology, Kakogawa City Hospital, Kakogawa, Hyogo, Japan

Takayuki Takahashi is currently at the Akasaka Hematology \& Oncology Clinic, Kobe, Japan Corresponding author: Takayuki Takahashi, Akasaka Hematology \& Oncology Clinic, 3-1-15 Yamada-chou, Nada-ku, Kobe 657-0064, Japan.

Received: May 18, 2020.

E-mail: takahashi.takayuki@shinkohp.or.jp

Revised: June 20, 2020.

Accepted: July 27, 2020.

Onlune Published: September 25, 2020

DOI:10.3960/jslrt.20020

Copyright $\odot 2020$ The Japanese Society for Lymphoreticular Tissue Research (cc) BY-NC-SA This work is licensed under a Creative Commons AttributionNonCommercial-ShareAlike 4.0 International License. 


\section{EXPERT'S COMMENT}

Okochi N et al. described a case of intestinal CD4+ T-cell lymphoma, not otherwise specified (NOS) with peritoneal lymphomatosis lacking large intestinal tumors. Findings of peritoneal lymphomatosis are rare in cases of intestinal T-cell lymphoma. Primary intestinal T/NK-cell lymphoproliferative disorders are classified into enteropathy-associated T-cell lymphoma, monomorphic epitheliotropic T-cell lymphoma (MEITL), indolent-type gastrointestinal T-cell lymphoma (GI-TCL), and NOS CD30+ anaplastic large cell lymphoma, nasal-type extranodal NK-cell lymphoma, and CD56+ NK-cell enteropathy without EBV infection. ${ }^{1}$ EATL mainly develops in Caucasians, and is composed of $\mathrm{CD} 3+, \mathrm{CD} 103+$, and CD30+/- large cell lymphoma, with approximately half of the patients having celiac disease. MEITL is frequent in East Asia and is unrelated to celiac disease, consisting of CD3+, CD103+, CD8+/-, CD56+/-, and EBER-negative cytotoxic T-cells. ${ }^{2}$ Indolent-type T-cell lymphoma is composed of multiple nodular lesions of small- to medium-sized CD4+ T-cell neoplasia, exhibiting similar findings to MALT lymphoma and long-term survival. ${ }^{3,4}$ GI-TCL, NOS is composed of $\mathrm{CD} 3+\mathrm{CD} 4+/-$, and CD8-/+ medium- to large-sized lymphoma cells, and patients have an advanced clinical stage with extraintestinal tumor cell invasion and a progressive clinical course. As patients with MEITL or GI-DLBCL often have accompanying perforation of the gastrointestinal wall, peritoneal dissemination of lymphoma cells may be observed. ${ }^{5}$ The current case exhibited peritoneal dissemination, probably due to perforation or mesenteric invasion of intestinal CD4+ TCL without tumor formation. GI-TCL, NOS is a provisional category of aggressive neoplasia and consists of frequent $\mathrm{CD} 4+$, occasional $\mathrm{CD} 8+$, and CD4-/ CD8- neoplastic T cells with frequent cytotoxic molecules. ${ }^{4}$ As there are few reports of GI-TCL, NOS, clear confirmative characteristics are required in the future. Peritoneal lymphomatosis is another special condition with or without symptoms of acute abdomen. ${ }^{6}$ Primary and secondary peritoneal lymphomatosis are occasionally observed in DLBCL, but they are rare in T/NK-cell lymphoma. Emergency laparotomy is occasionally required for high-grade GI lymphoma due to tumor perforation and peritoneal dissemination. When peritoneal T/NK-cell lymphoma is confirmed by cytological and histological examinations, the GI tract must be examined.

\section{REFERENCES}

1 van Vliet C, Spagnolo DV. T- and NK-cell lymphoproliferative disorders of the gastrointestinal tract: review and update. Pathology. 2020; 52 : 128-141.

2 Kikuma K, Yamada K, Nakamura S, et al. Detailed clinicopathological characteristics and possible lymphomagenesis of type II intestinal enteropathy-associated T-cell lymphoma in Japan. Hum Pathol. 2014; 45 : 1276-1284.

3 Kawamoto K, Nakamura S, Iwashita A, et al. Clinicopathological characteristics of primary gastric T-cell lymphoma. Histopathology. 2009; 55 : 641-653.

4 Soderquist CR, Bhagat G. Gastrointestinal T- and NK-cell lymphomas and indolent lymphoproliferative disorders. Semin Diagn Pathol. 2020; 37 : 11-23.

5 Tanaka H, Ambiru S, Nakamura S, et al. Successful diagnosis of type II enteropathy-associated T-cell lymphoma using flow cytometry and the cell block technique of celomic fluid manifesting as massive pyoid ascites that could not be diagnosed via emergency laparotomy. Intern Med. 2014; 53 : 129-133.

6 Das DK, Al-Juwaiser A, George SS, et al. Cytomorphological and immunocytochemical study of non-Hodgkin's lymphoma in pleural effusion and ascitic fluid. Cytopathology. 2007; 18 : 157-167

Morishige Takeshita MD

Department of Pathology Faculty of Medicine Fukuoka University

E-mail: m-take@adm.fukuoka-u.ac.jp 